\title{
Benchmark Study of Density Functionals for the Insertions of Olefin and Polar Monomers Catalyzed by $\alpha$-Diimine Palladium Complexes
}

\author{
Ce Liang ${ }^{1}$, Jimin Yang ${ }^{1}$, gen Luo $^{2}$, and Yi Luo ${ }^{1}$ \\ ${ }^{1}$ Dalian University of Technology \\ ${ }^{2}$ Anhui University
}

May 21, 2020

\begin{abstract}
The performance of a series of density functionals has been tested for the insertions of ethylene, methyl acrylate (MA), and vinyl bromide (VB) catalyzed by $\alpha$-diimine palladium complexes. Sixty-seven density functionals are screened, and the results are compared with available experimental data. Eleven hybrid functionals (M06, BHandH, mPW1PW91, HSEh1PBE, mPW3PBE, LC- $\omega$ PBE, mPW1PBE, PBE0, M06-HF, M06-2X, M05-2X) showed better performance in the insertions of both ethylene and MA, and could be therefore suitable for ethylene-MA copolymerization. Meanwhile, three GGA (PW91PW91, HCTH, HCTH407), two meta-GGA (TPSSTPSS, tHCTH), and ten hybrid functionals (M06, BHandH, TPSSh, B971, B98, B1B95, PBE0, M06-2X, tHCTHhyb, M05-2X) perform well in the ethylene-VB copolymerization. Besides, nine D3 or D3BJ augmented functionals are found to be suitable for both copolymerization systems. The D2 dispersion correction overestimated insertion energy barriers of these monomers and is unsuitable for such copolymerization. In addition, the double-zeta basis set is found to be sufficient for solvation single-point calculation of these systems.
\end{abstract}

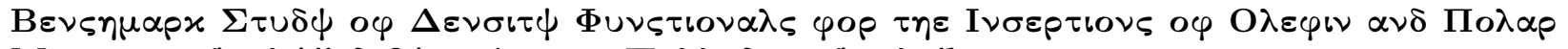

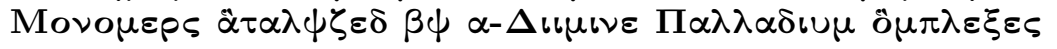

Ce Liang, ${ }^{+}$Jimin Yang, ${ }^{+}$Gen Luo, ${ }^{\S}$ Yi Luo ${ }^{+, *}$

${ }^{+}$State Key Laboratory of Fine Chemicals, School of Chemical Engineering, Dalian University of Technology, Dalian 116024, China

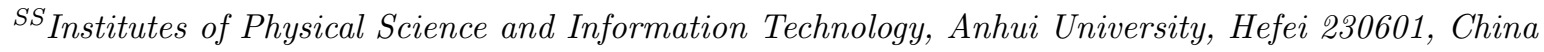

Abstract : The performance of a series of density functionals has been tested for the insertions of ethylene, methyl acrylate (MA), and vinyl bromide (VB) catalyzed by $\alpha$-diimine palladium complexes. Sixty-seven density functionals are screened, and the results are compared with available experimental data. Eleven hybrid functionals (M06, BHandH, mPW1PW91, HSEh1PBE, mPW3PBE, LC- $\omega$ PBE, mPW1PBE, PBE0, M06-HF, M06-2X, M05-2X) showed better performance in the insertions of both ethylene and MA, and could be therefore suitable for ethylene-MA copolymerization. Meanwhile, three GGA (PW91PW91, HCTH, HCTH407), twometa -GGA (TPSSTPSS, tHCTH), and ten hybrid functionals (M06, BHandH, TPSSh, B971, B98, B1B95, PBE0, M06-2X, tHCTHhyb, M05-2X) perform well in ethylene-VB copolymerization. Besides, nine D3 or D3BJ augmented functionals are found to be suitable for both copolymerization systems. The D2 dispersion correction overestimated insertion energy barriers of these monomers and is unsuitable for such copolymerization. In addition, the double-zeta basis set is found to be sufficient for solvation single-point calculation of these systems.

Keywords: Benchmark of DFT, Diimine palladium, Olefin insertion, Polar monomer, Cross-coupling

\section{INTRODUCTION}


Diimine ligated late-transition-metal complexes are capable of catalyzing not only Heck, Suzuki, and Hiyama coupling reactions, ${ }^{1}$ but also (co)polymerization of olefins. ${ }^{2,3}$ In this context, the catalytic copolymerization of ethylene and polar monomers is a straightforward and atom-economic method to synthesize functionalized polyolefin materials of industrial importance. ${ }^{3-15}$ However, there are many challenges in these copolymerization systems, such as low reaction activity and low polar monomer incorporation. It is obvious that further study of such copolymerization mechanisms at molecular and electronic levels is highly required to develop more efficient copolymerization systems. In this instance, the issues of large size of such catalytic systems and computational resources could rule the highly accurateab-initio wavefunction based methods out. Density functional theory (DFT) offered an ideal solution to study the mechanism of such copolymerization systems. ${ }^{16-22}$ However, many density functionals have no broad application range, and the application of individual functionals to specific systems needs performance test according to either experimental or ab-initio results. It is therefore necessary to choose a suitable and accurate DFT method to deal with the system of interest. Given the importance of developing the ethylene/polar monomer copolymerization system, it is worth of performing a benchmark study of DFT functionals for the mechanistic study of such copolymerizations catalyzed by late transition metal complexes.

In 2006, Martin et al. ${ }^{23}$ performed a benchmark study on C-C and C-H activations by bare palladium atom, and found thatmeta -GGA functionals have good performances. Recently, Checink et al. conducted thermochemical benchmark set of closed-shell metal organic system including carbene-ligated Pd complex and presented a series of applicable functionals. It is noteworthy that two commonly used functionals, BP86(D3) and B3LYP(D3) had poor performance. ${ }^{24}$ Schreckenbach et al. carried out a benchmark study on thermodynamics of hydrocarbon isomerization and olefin monomer insertion, but without consideration of metal catalysis. They found that the dispersion augmented BPBE, PBEPBE, and B3LYP functionals showed better performance compared with other functional investigated. ${ }^{25}$ Maron et al. studied the solvation and dispersion effects on group 3 and 4 metallocene-catalyzed propylene coordination and insertion reactions. It was revealed that dispersion effects generally overestimate the stability of olefin complexes and B3PW91 functional performed well in a comprehensive consideration of computational cost and precision. ${ }^{26}$ Although significant progress has been achieved in this field, as far as we are aware, the benchmark study of theoretical methods for olefin copolymerization systems catalyzed by late transition metal has not been reported to date. In the present work, a benchmark study on the insertion of three monomers, viz. ethylene, methyl acrylate (MA) and vinyl bromide (VB) into ( $\alpha$-diimine)Pd-Me bond has been conducted, respectively, where there is available experimental data (Table 1). ${ }^{4,27}$ By screening 67 functional methods, a series of functionals have been found to perform well in the prediction of insertion activation energies of these monomers, and their intersection could be suitable for dealing with such copolymerization systems. It is also found that double-zeta basis sets are sufficient for solvation single-point calculations with SMD or CPCM models.

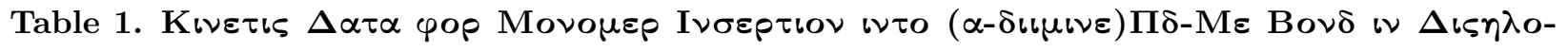

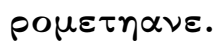

\section{COMPUTATIONAL METHODS AND STRATEGIES}

All calculations were performed with the Gaussian 16 program. ${ }^{28}$ Various types of functionals, such as GGA, meta -GGA, hybrid, and functionals with semi-empirical dispersion correction (D2 ${ }^{29}$, D $3^{30}$, and D3BJ ${ }^{31}$ ) were used for geometry optimizations. In these geometrical optimizations, the LANL2DZ basis set $^{32}$ together with effective core potential (ECP) was used for $\mathrm{Pd}$ and $\mathrm{Br}$ atoms and the 6-31G(d) basis set was used for $\mathrm{C}, \mathrm{H}, \mathrm{O}$ and $\mathrm{N}$ atoms. In order to verify the optimized stationary points and obtain the thermodynamic data at the actual reaction temperature, vibration frequency analyses were conducted at the same level of theory. Based on each optimized structure, single point calculation was performed with the corresponding DFT functional used for its geometrical optimization. In these single-point calculations, larger basis sets, viz., the SDD and associated effective core potential ${ }^{33}$ for $\mathrm{Pd}$ and $\mathrm{Br}$ atoms and the triplezeta $6-311 \mathrm{G}(\mathrm{d}, \mathrm{p})$ basis set for the remaining atoms, are applied, and the solvation effect of dichloromethane generally used in such copolymerization systems was considered by using SMD solvation model. ${ }^{34}$ The Gibbs free energy in solution, including gas-phase free energy correction, was used for discussion, unless otherwise 
specified. The functionals were screened by comparing the experimentally measured activation energy with the corresponding calculated value. For a comparison of the effect of solvation models, CPCM model ${ }^{34}$ was also considered for some calculations. Three model chemistries used for fitting the parameters of SMD solvation model by Truhlar et al. were selected in the solvation calculations, namely, M05-2X/6-31G(d), M05-2X/6-31+G(d,p), and M05-2X/cc-pVTZ. ${ }^{35}$ Similarly, in the CPCM solvation calculations, the two model chemistries of PBE0/6-31+G(d) and PBE0/6-31+G(d,p) were utilized, which was previously used for testing the accuracy of CPCM solvation model by Barone et al. ${ }^{34}$

The calculation strategy is shown in Figure 1. Based on the catalytic active species, the energy barrier for ethylene insertion is calculated and compared with the corresponding experimental value to screen the density functionals. Then, using the functionals with better performance, the insertion energy barrier of the two polar monomers were calculated, respectively. Compared with the corresponding experimental values, the functionals with better performance for the insertions of both ethylene and the polar monomer are considered to be suitable for the calculation of copolymerization system. According to the obtained results, single-point energy was further calculated at the specific theoretical levels together with the SMD and CPCM models, respectively, to evaluate the effect of solvation models. The density functional, which produces less than an energy barrier error of $1.0 \mathrm{kcal} / \mathrm{mol}$ compared with corresponding experimental values, is considered to be better for the calculations of such copolymerization systems.

Figure 1 . Flow chart of calculation strategy.

\section{RESULTS AND DISCUSSION}

Forty-one density functionals shown in Table 2 has been tested, including eight GGA, three meta-GGA, and thirty hybrid functionals. Among them, additional D2, D3, and D3BJ dispersion corrections were also considered for five (B97D functional employing D2 correction as a default in the program), thirteen, and eight functionals (Table 2), respectively. Therefore, the total 67 methods have been applied.

Table 2. All Tested Density Functionals ${ }^{1 \text { ) }}$

\begin{tabular}{|c|c|c|c|c|c|}
\hline & functional & D2 & D3 & D3BJ & Reference \\
\hline \multirow[t]{8}{*}{$\overline{\text { GGA }}$} & BP86 & {$[?]$} & {$[?]$} & & 36,37 \\
\hline & B97D & {$[?]$} & & & 38 \\
\hline & PW91PW91 & {$[?]$} & {$[?]$} & {$[?]$} & 3940 \\
\hline & PBEPBE & & & & \\
\hline & НCTH & & & & $41,42,43$ \\
\hline & НCTH407 & & & & $41,42,43$ \\
\hline & BLYP & {$[?]$} & {$[?]$} & {$[?]$} & 36 \\
\hline & BPBE & & {$[?]$} & [?] & 36,40 \\
\hline \multirow[t]{3}{*}{ Meta-GGA } & TPSSTPSS & {$[?]$} & {$[?]$} & {$[?]$} & 44 \\
\hline & M06L & & {$[?]$} & & 45 \\
\hline & tHCTH & & & & 46 \\
\hline \multirow[t]{12}{*}{ Hybrid } & M06 & & & & 47 \\
\hline & B3LYP & {$[?]$} & {$[?]$} & {$[?]$} & 48,49 \\
\hline & B3P86 & & & & 50 \\
\hline & B3PW91 & & {$[?]$} & {$[?]$} & 51 \\
\hline & B1B95 & & & & 52 \\
\hline & mPW1PW91 & & & & 53,54 \\
\hline & mPW1LYP & & & & 53,55 \\
\hline & mPW1PBE & & & & 40,53 \\
\hline & mPW3PBE & & & & 40,53 \\
\hline & B98 & & & & 54 \\
\hline & B971 & & & & 41 \\
\hline & B972 & & & & 56 \\
\hline
\end{tabular}




\begin{tabular}{|c|c|c|c|c|}
\hline functional & D2 & D3 & D3BJ & Reference \\
\hline PBE0 & & {$[?]$} & {$[?]$} & 57 \\
\hline B1LYP & & & & 58 \\
\hline O3LYP & & & & 59 \\
\hline M062X & & {$[?]$} & & 47 \\
\hline tHCTHhyb & & & & 46 \\
\hline HSEh1PBE & & & & 60,61 \\
\hline$\omega B 97 X D$ & & & & 62 \\
\hline$\omega \mathrm{B} 97$ & & & & 63 \\
\hline TPSSh & & & & 44,64 \\
\hline X3LYP & & & & 65 \\
\hline $\mathrm{LC}-\omega \mathrm{PBE}$ & & {$[?]$} & & 66,67 \\
\hline CAM-B3LYP & & & {$[?]$} & 68 \\
\hline BMK & & & & 69 \\
\hline BHandH & & & & 70 \\
\hline M06-HF & & & & 71,72 \\
\hline BHandHLYP & & & & 70 \\
\hline M05-2X & & {$[?]$} & & 73 \\
\hline M05 & & {$[?]$} & & 74 \\
\hline
\end{tabular}

The mark "[?]" indicates that the functional includes the corresponding dispersion correction, respectively.

\section{Functional screening for copolymerization systems}

Firstly, functionals in Table 2 are used to calculate the energy barrier for ethylene insertion into the Pd-Me bond of initial active species (Table 1). The obtained results are compared with the experimental kinetic data (free energy barrier of $17.2 \mathrm{kcal} / \mathrm{mol}$ at the temperature of $243.6 \mathrm{~K}$ ). As shown in Figure 2, B97D functional has the largest positive deviation of $3.8 \mathrm{kcal} / \mathrm{mol}$ and M05 has the largest negative deviation of $-2.3 \mathrm{kcal} / \mathrm{mol}$. On the other hand, the functionals with semi-empirical dispersion correction (D2, D3, or D3BJ) overestimate the ethylene insertion barrier except for B3PW91-D3, PBE0-D3, and M05-D3 (Figure 3). A similar situation was reported by Maron et al. that dispersion correction could overestimate the energy barrier for propene insertion into early transition metal complexes. ${ }^{26}$ To get an image about the effect of dispersion correction on the calculated energy barrier, the absolute error of the barrier for ethylene insertion calculated by individual functional without and with available additional dispersion correction to the functional has been compared (Figure S1). It is found that the TPSSTPSS (meta-GGA) functional produced an error of $0.9 \mathrm{kcal} / \mathrm{mol}$. In the case of dispersion correction to this functional, viz., TPSSTPSS-D2, TPSSTPSS-D3, and TPSSTPSSD3BJ, the errors are 3.0,1.7, and $1.6 \mathrm{kcal} / \mathrm{mol}$, respectively, suggesting an overestimation of insertion energy barrier by the dispersion correction. Although some dispersion correction augmented functionals produced an error of less than $1.0 \mathrm{kca} / \mathrm{mol}$, such an overestimation caused by dispersion correction was also observed for BPBE, BP86, PBEPBE, BLYP, and B3LYP functionals, compared with corresponding functionals without dispersion correction. There are 38 methods with better performance (energy barrier error of less than 1.0 $\mathrm{kcal} / \mathrm{mol}$ ) for the ethylene insertion. These functionals includes 6 GGA, 2 meta -GGA, 20 hybrid types of functionals, PBEPBE (D2), 5 functionals with D3 dispersion correction and 4 functionals with D3BJ dispersion correction (Figures 2 and 3 ). 


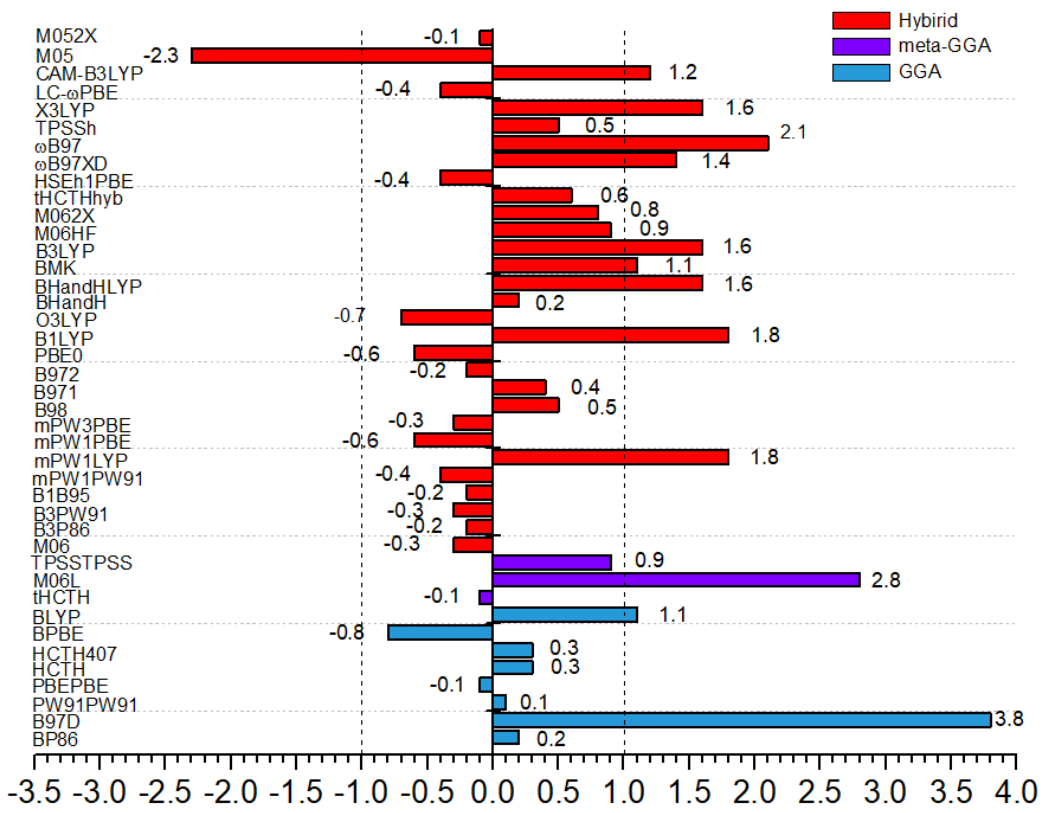

Figure 2. The error bar of calculated energy barriers $\left(\Delta G^{[?]}, \mathrm{kcal} / \mathrm{mol}\right)$ for ethylene insertion in comparison with the experimental value $(17.2 \mathrm{kcal} / \mathrm{mol}) .{ }^{4}$ The positive error value means overestimation of energy barrier, and the negative error means underestimation. The same is true for the following figures.

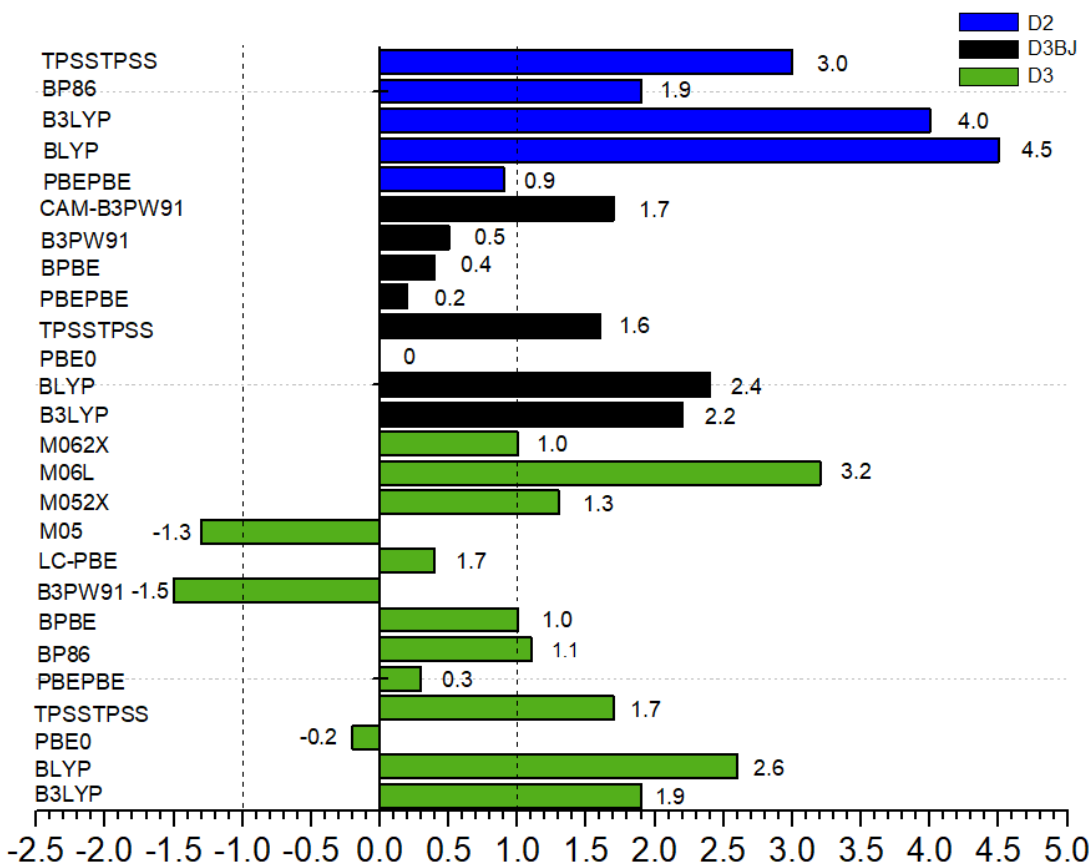

Figure 3. The error bar of calculated energy barriers $\left(\Delta G^{[?]}, \mathrm{kcal} / \mathrm{mol}\right)$ for ethylene insertion in comparison with the experimental value $(17.2 \mathrm{kcal} / \mathrm{mol}) .{ }^{4}$ The functionals shown include available semi-empirical dispersion corrections. 
By using the 38 methods with better performance for ethylene insertion, the insertion energy barriers of two polar monomers, MA and VB, have been further calculated (Table 1, Figures 4 and 5). The reported kinetic data for MA insertion are $\Delta H^{[?]}=12.1 \pm 1.4 \mathrm{kcal} / \mathrm{mol}$ and $\Delta S^{[?]}=-14.1 \pm 7.0 \mathrm{cal} / \mathrm{K}[?] \mathrm{mol}{ }^{27}$ Due to the large range of entropy change $(+-7.0 \mathrm{cal} / \mathrm{K}[?] \mathrm{mol})$, the corresponding Gibbs free energy could vary significantly from the central value and is unsuitable for functional screen. Therefore, the enthalpy value for MA insertion was used instead. The reported kinetic data for VB insertion are $\Delta H^{[?]}=11.9 \pm 0.1$ $\mathrm{kcal} / \mathrm{mol}, \Delta S^{[?]}=-16.8 \pm 0.1 \mathrm{cal} / \mathrm{K}[?] \mathrm{mol}$ at $236.5 \mathrm{~K}$, and the corresponding $\Delta G^{\left[{ }^{[?]}\right.}$ is estimated to be 15.9 $\pm 0.1 \mathrm{kcal} / \mathrm{mol}$.

The calculated energy barriers for MA insertion are shown in Figure 4. It is found that 11 hybrid functionals, 5 functionals with D3 correction and 4 functionals with D3BJ correction showed an error of less than 1.0 $\mathrm{kcal} / \mathrm{mol}$. That is, these methods behave better for the calculation of $\alpha$-diimine palladium catalyzed ethyleneMA copolymerization. All the GGA and meta -GGA functionals produced an error of more than $1.0 \mathrm{kcal} / \mathrm{mol}$. Among these functionals, B1B95 gives the worst result ( $\Delta H^{[?]}$ of 16.8 vs $\left.12.1 \pm 1.4 \mathrm{kcal} / \mathrm{mol}\right)$. As a result, GGA and meta-GGA functionals produced relatively large errors for ethylene-MA copolymerization, and the inclusion of D3 or D3BJ dispersion correction tend to reduce the energy barrier for MA insertion compared with their original form without such corrections.

Unlike the case of ethylene/MA copolymerization, GGA and meta-GGA functionals such as PBEPBE, PW91PW91, HCTH407, HCTH, TPSSTPSS and tHCTH showed good performance in ethylene/VB copolymerization (Figure 5). Moreover, several functionals with D3 and D3BJ correction and 10 hybrid functionals have better performance. Functional TPSSTPSS-D2 gives the worst result with the energy barrier value up to $18.1 \mathrm{kcal} / \mathrm{mol}$ (experimental value of $15.9 \pm 0.1 \mathrm{kcal} / \mathrm{mol}$ ). In addition, the functional such as BPBE with D3 or D3BJ dispersion correction generally overestimated energy barrier compared with its original form (without the dispersion correction).

Schreckenbach et al. found that the functionals, viz., BPBE, PBEPBE, and B3LYP, augmented by D3BJ dispersion correction showed good performance in the calculations of hydrocarbon isomerization and metalfree olefin insertion model system. ${ }^{25}$ For the two copolymerization systems in this study, BPBE(D3BJ) and PBEPBE(D3BJ) also performs well. In a previously reported organocatalytic systems ${ }^{75}$ the M06-2X functional with implicit dispersion correction tended to give high accuracy. This is also found in the current work. However, the commonly used B3LYP functional showed relatively poor performance for ethylene insertion regardless of the inclusion of dispersion correction (with errors of $1.6 \mathrm{kcal} / \mathrm{mol}$ for B3LYP, $2.2 \mathrm{kcal} / \mathrm{mol}$ for B3LYP-D3BJ, and $4.0 \mathrm{kcal} / \mathrm{mol}$ for B3LYP-D2). The hybrid functionals M06-2X and PBE0 have also good performance without dispersion correction. Besides, D2 dispersion correction significantly overestimated the insertion energy barriers of these monomers (Figures 3-5). Therefore, D2 dispersion correction could be unsuitable for such polymerization systems. 


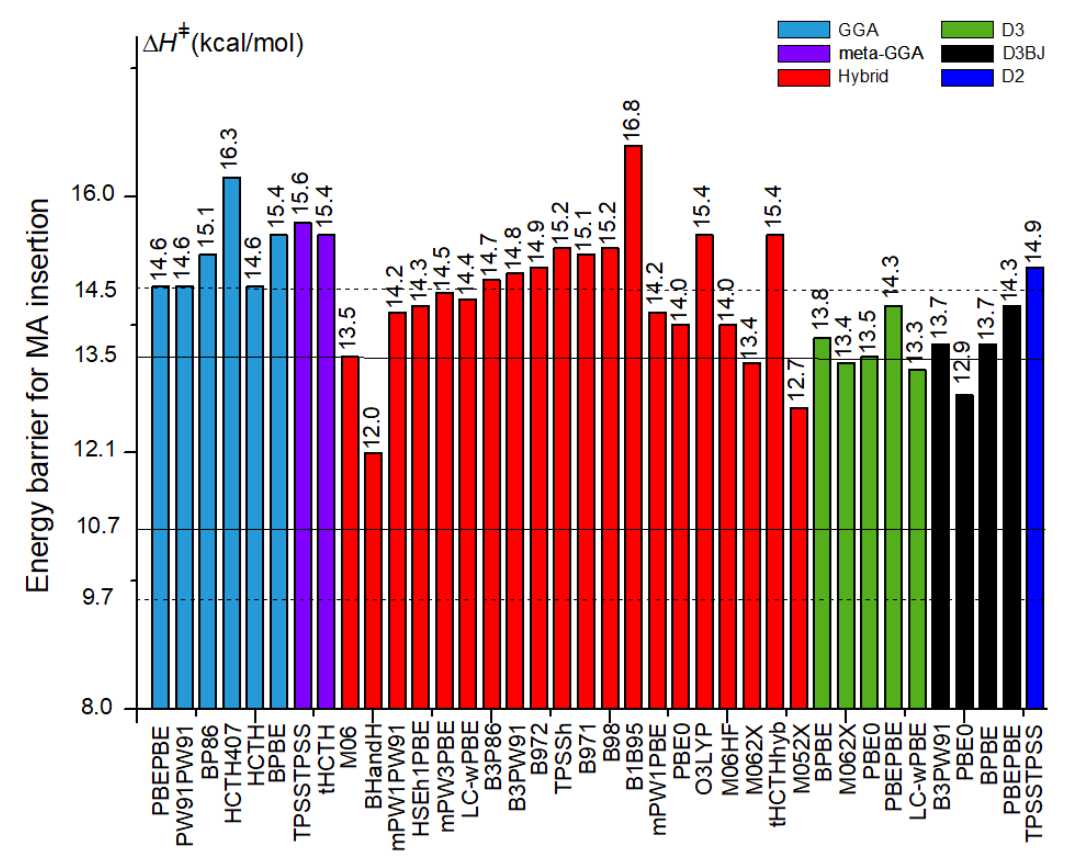

Figure 4. The energy barriers $\left(\Delta H^{[?]}, \mathrm{kcal} / \mathrm{mol}\right)$ for MA insertion calculated by various functionals.

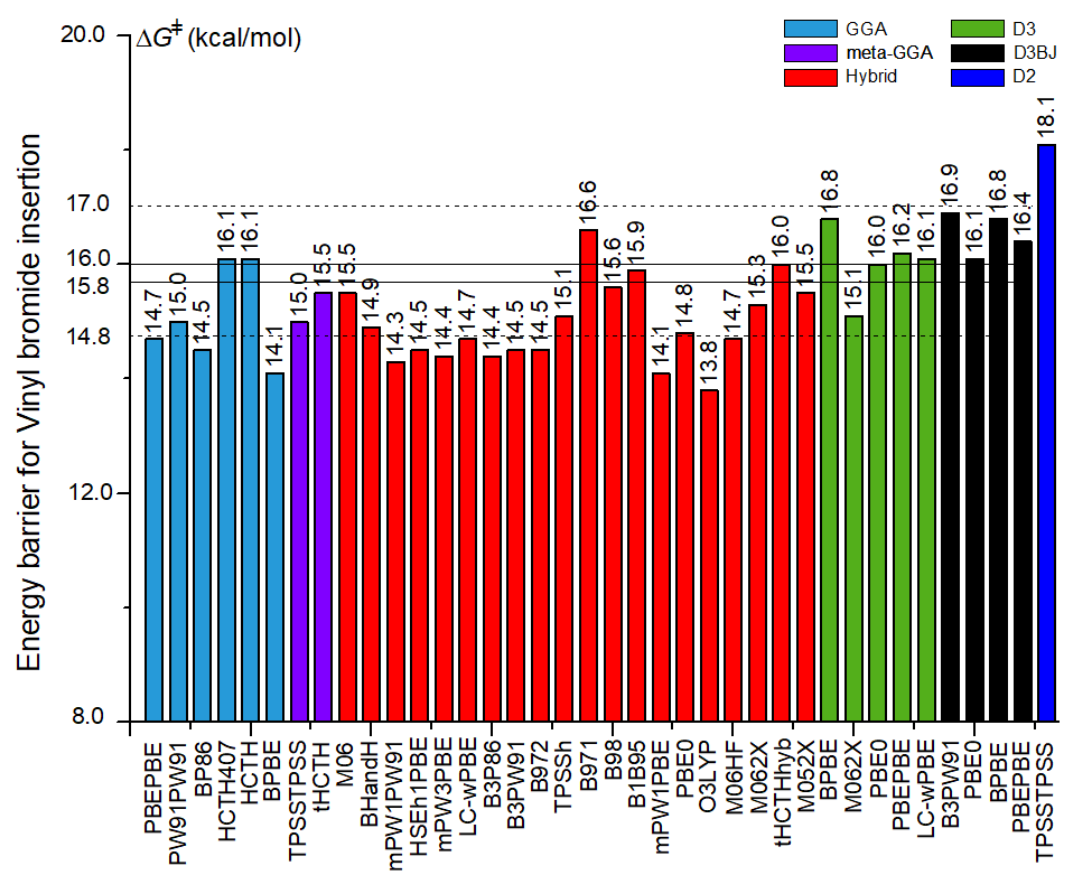

Figure 5. The energy barriers $\left(\Delta G^{[?]}, \mathrm{kcal} / \mathrm{mol}\right)$ for the VB insertion calculated by various functionals. Effects of solvation model and associated methods

On the basis of screened density functionals discussed above, the effect of solvation models together with specific theoretical levels have been further investigated. Solvation single-point calculations were performed 
with consideration of two common solvation models (SMD and CPCM) at the theoretical level originally used for fitting the parameters or recommended for the corresponding solvation model. ${ }^{34,35}$

\section{SMD model}

All tested functionals having good performance (error of $<1.0 \mathrm{kcal} / \mathrm{mol}$ ) for ethylene insertion are collected in Table 3. As shown in this table, at the level of M05-2X(SMD)/6-31G(d)[?]SDD, all functionals except HCTH407, HCTH, and BPBE(D3BJ) performs well for ethylene insertion using the SMD model. It is noted that double-zeta basis set is sufficient to obtain accurate result and there is (usually) no need to use the triple-zeta basis 6-311G(d,p) shown above. For copolymerization of ethylene and MA, all the selected functionals give relatively accurate insertion energy barriers. In addition, the M06 functional shows good performance, which may be due to the fact that this functional takes into account the weak interactions. This is also reflected in both the functional with implicit weak interactions and that with the correction of long-range interactions. For copolymerization of ethylene and VB, the functional BPBE(D3) shows good performance. All tested hybrid functionals gave good results except M06, TPSSh, and M06-HF. The GGA and meta -GGA functional underestimate the barrier and have relatively poor performance (error of $>1.3$ $\mathrm{kcal} / \mathrm{mol}$ ). It is also found that the error derived from PBEPBE functional is still relatively large after adding D3 and D3BJ dispersion correction (error of $>1.5 \mathrm{kcal} / \mathrm{mol}$ ). For the functionals with dispersion correction, viz. PBE0(D3), LC- $\omega$ PBE(D3), B3PW91(D3BJ), and PBE0(D3BJ), perform well. Therefore, the M05-2X functional together with double-zeta basis set 6-31G(d) (SDD for metal atom) can be used for the solvation single-point calculation on the geometries optimized by most functionals tested for such copolymerization systems. To further confirm this, larger basis sets $(6-31+\mathrm{G}(\mathrm{d}, \mathrm{p})$ and cc-pVTZ) were also considered for the non-metal atoms, respectively. The results are almost the same as that from M05-2X/6-31G(d) level. (Figure S2-S7 in SI).

\section{CPCM model}

To test the performance of CPCM solvation model in such copolymerization systems, single-point solvation calculations have been performed at the level of PBE0/6-31+G(d), which was shown to be suitable for CPCM model. ${ }^{34}$ As shown in Table 3, in the case of ethylene insertion, all the tested functionals underestimated the reaction energy barrier, among which the hybrid M06-HF performs the worst with an error of 2.3 $\mathrm{kcal} / \mathrm{mol}$. For MA insertion, all the functionals shown in Table 3 perform well. In the case of VB insertion, like the case of SMD model, GGA andmeta -GGA functionals underestimated the reaction energy barrier with errors of larger than $1.0 \mathrm{kcal} / \mathrm{mol}$. The hybrid functional LC- $\omega \mathrm{PBE}$ with D3 dispersion correction overestimated the barrier $(17.9 v s 15.9 \pm 0.1 \mathrm{kcal} / \mathrm{mol})$. This can be ascribed to that the functional LC- $\omega \mathrm{PBE}$ itself considered the long-range interaction, and further D3 dispersion correction may lead to overcorrection. The hybrid functional BHandH also overestimated the energy barrier $(17.3 \mathrm{kcal} / \mathrm{mol})$. When larger basis set $6-31+\mathrm{G}(\mathrm{d}, \mathrm{p})$ was used for the non-metal atoms, similar results was obtained (Table S1). Therefore, with the CPCM solvent model, the lower theoretical level of $6-31+\mathrm{G}(\mathrm{d})$ for single-point calculation is sufficient to obtain accurate energy barriers in such copolymerization system. It is noteworthy that, although some functionals produced errors of $>1.0 \mathrm{kcal} / \mathrm{mol}$, the errors are not so large $(<3 \mathrm{kcal} / \mathrm{mol})$, suggesting a wide range of functionals suitable for dealing with such ( $\alpha$-diimine)Pd-catalyzed copolymerizations.

Table 3. The Calculated Energy Barriers (kcal/mol) in Solution for the Monomer Insertion by Using the SMD and CPCM Solvation Models ${ }^{1)}$

\begin{tabular}{|c|c|c|c|c|c|c|c|}
\hline $\begin{array}{l}\text { DFT } \\
\text { Method }\end{array}$ & $\begin{array}{l}\text { DFT } \\
\text { Method }\end{array}$ & $\begin{array}{l}\text { M05- } \\
2 \mathrm{X} / 6- \\
31 \mathrm{G}(\mathrm{d})^{2)}, \\
\mathrm{SMD}\end{array}$ & $\begin{array}{l}\text { M05- } \\
2 \mathrm{X} / 6- \\
31 \mathrm{G}(\mathrm{d})^{2)}, \\
\mathrm{SMD}\end{array}$ & $\begin{array}{l}\text { M05- } \\
2 \mathrm{X} / 6- \\
31 \mathrm{G}(\mathrm{d})^{2)}, \\
\text { SMD }\end{array}$ & $\begin{array}{l}\text { PBE0/6- } \\
31+\mathrm{G}(\mathrm{d}) \\
\text { 2), CPCM }\end{array}$ & $\begin{array}{l}\text { PBE0/6- } \\
31+\mathrm{G}(\mathrm{d}) \\
\text { 2), CPCM }\end{array}$ & $\begin{array}{l}\text { PBE0/6- } \\
31+\mathrm{G}(\mathrm{d}) \\
\text { 2), } \mathrm{CPCM}\end{array}$ \\
\hline & & $\begin{array}{l}\mathrm{Et}^{3)} \\
\Delta G^{[?]}\end{array}$ & MA $\Delta H^{[?]}$ & $\mathrm{VB} \Delta G^{[?]}$ & Et $\Delta \mathrm{G}^{[?]}$ & MA $\Delta H^{[?]}$ & $\mathrm{VB} \Delta G^{[?]}$ \\
\hline GGA & PW91PW91 & 16.6 & - & 14.0 & 16.4 & - & 14.5 \\
\hline
\end{tabular}




\begin{tabular}{|c|c|c|c|c|c|c|c|}
\hline $\begin{array}{l}\text { DFT } \\
\text { Method } \\
\end{array}$ & $\begin{array}{l}\text { DFT } \\
\text { Method }\end{array}$ & $\begin{array}{l}\text { M05- } \\
2 \mathrm{X} / 6- \\
31 \mathrm{G}(\mathrm{d})^{2)} \\
\text { SMD }\end{array}$ & $\begin{array}{l}\text { M05- } \\
2 \mathrm{X} / 6- \\
31 \mathrm{G}(\mathrm{d})^{2)} \\
\text { SMD }\end{array}$ & $\begin{array}{l}\text { M05- } \\
2 \mathrm{X} / 6- \\
31 \mathrm{G}(\mathrm{d})^{2)}, \\
\text { SMD }\end{array}$ & $\begin{array}{l}\mathrm{PBE} 0 / 6- \\
31+\mathrm{G}(\mathrm{d}) \\
2), \mathrm{CPCM}\end{array}$ & $\begin{array}{l}\mathrm{PBE} 0 / 6- \\
31+\mathrm{G}(\mathrm{d}) \\
{ }^{2)}, \mathrm{CPCM}\end{array}$ & $\begin{array}{l}\mathrm{PBE} 0 / 6- \\
31+\mathrm{G}(\mathrm{d}) \\
\text { 2), } \mathrm{CPCM}\end{array}$ \\
\hline & HCTH407 & 16.1 & - & - & 16.0 & - & - \\
\hline & $\mathrm{HCTH}$ & 16.1 & - & - & 16.0 & - & - \\
\hline \multirow{2}{*}{$\begin{array}{l}\text { meta- } \\
\text { GGA }\end{array}$} & tHCTH & 16.3 & - & 14.3 & 16.2 & - & 14.5 \\
\hline & TPSSTPSS & 16.4 & - & 13.9 & 16.4 & - & 14.4 \\
\hline \multirow[t]{15}{*}{ Hybrid } & $\begin{array}{l}\text { M06 } \\
\text { BHandH }\end{array}$ & 17.517 .8 & 13.613 .6 & 14.415 .5 & 16.416 .7 & 13.614 .1 & 14.817 .3 \\
\hline & mPW1PW91 & 17.2 & 13.3 & - & 16.5 & 13.1 & - \\
\hline & HSEh1PBE & 17.0 & 13.4 & - & 16.3 & 13.6 & - \\
\hline & mPW3PBE & 17.0 & 13.3 & - & 16.5 & 13.6 & - \\
\hline & LC- $\omega \mathrm{PBE}$ & 17.6 & 14.2 & - & 16.5 & 14.1 & - \\
\hline & TPSSh & 16.6 & - & 14.0 & 16.4 & - & 14.3 \\
\hline & B971 & 17.0 & - & 16.1 & 16.4 & - & 15.8 \\
\hline & B98 & 17.1 & - & 15.4 & 16.5 & - & 15.1 \\
\hline & B1B95 & 17.2 & - & 16.1 & 16.6 & - & 16.1 \\
\hline & mPW1PBE & 17.1 & 13.4 & - & 16.5 & 13.6 & - \\
\hline & PBE0 & 17.0 & 13.0 & - & 16.4 & 13.6 & - \\
\hline & M06-HF & 17.2 & 12.7 & 13.8 & 14.9 & - & - \\
\hline & M06-2X & 17.8 & 13.5 & 14.9 & 16.3 & 12.4 & 14.3 \\
\hline & tHCTHhyb & 17.1 & - & 15.6 & 16.6 & - & 15.3 \\
\hline & M05-2X & 17.9 & 12.9 & 16.0 & 16.5 & 11.8 & 15.9 \\
\hline \multirow[t]{5}{*}{ DFT-D3 } & $\mathrm{BPBE}$ & 16.2 & 12.4 & 14.9 & 16.1 & - & - \\
\hline & M06-2X & 17.9 & 13.6 & 14.8 & 16.6 & 12.5 & 14.3 \\
\hline & PBE0 & 17.0 & 13.5 & 15.3 & 16.1 & - & - \\
\hline & PBEPBE & 16.4 & 13.1 & 13.8 & 16.1 & - & - \\
\hline & LC- $\omega \mathrm{PBE}$ & 17.5 & 13.6 & 16.4 & 16.5 & 12.6 & 17.9 \\
\hline \multirow{4}{*}{$\begin{array}{l}\text { DFT- } \\
\text { D3BJ }\end{array}$} & B3PW91 & 16.8 & 13.5 & 15.4 & 16.1 & - & - \\
\hline & PBE0 & 17.1 & 13.0 & 15.1 & 16.5 & 12.7 & 16.0 \\
\hline & BPBE & 15.7 & - & - & 15.7 & - & - \\
\hline & PBEPBE & 16.2 & 12.8 & 14.2 & 16.1 & - & - \\
\hline Experiment $^{4,27}$ & Experiment ${ }^{4,27}$ & 17.2 & $12.1 \pm 1.4$ & $15.9 \pm 0.1$ & 17.2 & $12.1 \pm 1.4$ & $15.9 \pm 0.1$ \\
\hline
\end{tabular}

1) The symbol "-" denotes that the corresponding functional produced larger error for corresponding monomer or ethylene insertion and was not considered here for the comparison of the effect of solvation models on copolymerizations.

2) The SDD basis set and associated effective core potential was used for Pd atom, while the corresponding full-electron basis set was used for non-metal atoms.

3) Et: ethylene; MA: methyl acrylate; VB: vinyl bromide.

\section{CONCULUSION}

The benchmark of DFT for $\alpha$-diimine palladium catalyzed insertions of ethylene, methyl acrylate, and vinyl bromide has been studied, during which sixty-seven functional methods have been screened on the basis of experimental kinetic data. 
For the ethylene/methyl acrylate copolymerization featuring the insertions of the both monomers, when the single-point calculation is performed at the level of M05-2X(SMD)/6-31G(d)[?]SDD, 11 hybrid functionals

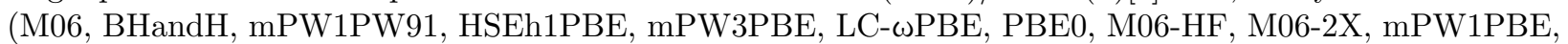
M05-2X), 5 functionals with D3 dispersion correction (BPBE-D3, M062X-D3, PBE0-D3, PBEPBE-D3, LC$\omega$ PBE-D3), and 3 functionals with D3BJ dispersion correction (B3PW91-D3BJ, PBE0-D3BJ, PBEPBED3BJ) used for geometrical optimization could give better results compared with other tested functionals. However, GGA and some meta-GGA functionals showed poor performance in the geometry optimization with respect to calculated free energy barrier in solution (SMD model). The hybrid functional B1B95 produced the worst result. When the CPCM model is applied at the level of PBE0(CPCM)/6-31+G(d)[?]SDD, the aforementioned 11 hybrid functionals except M06-HF also showed good performance. At this singlepoint calculation level, the M06, M062X-D3, LC- $\omega$ PBE-D3, and PBE0-D3BJ geometries can also produce accurate free energy barrier in solution.

For the copolymerization ethylene and vinyl bromide, when the single-point calculation is carried out at the level of PBE0(CPCM)/6-31+G(d)[?]SDD, the tested hybrid functionals except BHandH, TPSSh, and M06$2 \mathrm{X}$ perform well. However, the GGA and meta -GGA functionals have poor performance, like the case of ethylene/MA copolymerization. And, LC- $\omega$ PBE-D3 overestimated the energy barrier of vinyl bromide insertion, possibly due to the over-correction of dispersion force. At the level of M05-2X(SMD)/6-31G(d)[?]SDD for single-point calculations, the GGA and meta -GGA still have poor performance. The dispersion correction augmented PBEPBE functional underestimated the barrier and showed worst performance among the tested functionals. The hybrid functionals except M06, TPSSh, and M06-HF perform well at this level.

It is noted that the performance of the common B3LYP functional with or without dispersion is not ideal in these systems. Compared with SMD solvation model, CPCM model slightly underestimated the energy barrier of ethylene insertion, but it is still within the acceptable error range.

\section{ASSOCIATED CONTENT}

Supporting Information

Additional table and figures showing the effect of dispersion corrections on the error of ethylene insertion energy barrier and monomer insertion energy barriers calculated at various levels together with SMD and CPCM solvation models as well as Cartesian coordinates of optimized stationary points. The Supporting Information is available free of charge on the ACS Publications website.

\section{AUTHOR INFORMATION}

Corresponding Author

* Y.L. (luoyi@dlut.edu.cn)

Notes The authors declare no competing financial interest.

\section{ACKNOWLEDGEMENT}

We are grateful for the financial support provided by the NSFC (21674014, U1862115) and the Fundamental Research Funds for the Central Universities (DUT18GJ201). The authors also thank the Network and Information Center of Dalian University of Technology for part of the computational resources.

\section{REFERENCES}

1. Domin, D.; Benito-Garagorri, D.; Mereiter, K.; Fröhlich, J.; Kirchner, K., Synthesis and Reactivity of Palladium and Nickel $\beta$-Diimine Complexes: Application as Catalysts for Heck, Suzuki, and Hiyama Coupling Reactions. Organometallics 2005, 24, 3957-3965.

2. Nakamura, A.; Ito, S.; Nozaki, K. Coordination-Insertion Copolymerization of Fundamental Polar Monomers. Chem. Rev.2009, 109 , 5215-5244. 
3. Chen, C., Designing catalysts for olefin polymerization and copolymerization: beyond electronic and steric tuning. Nat. Rev. Chem. 2018, 2, 6-14.

4. Johnson, L. K.; Killian, C. M.; Brookhart, M., New Pd(II)- and Ni(II)-Based Catalysts for Polymerization of Ethylene and .alpha.-Olefins. J. Am. Chem. Soc. 1995, 117, 6414-6415.

5. Johnson, L. K.; Mecking, S.; Brookhart, M., Copolymerization of Ethylene and Propylene with Functionalized Vinyl Monomers by Palladium(II) Catalysts. J. Am. Chem. Soc. 1996 ,118, 267-268.

6. Drent, E.; van Dijk, R.; van Ginkel, R.; van Oort, B.; Pugh, R. I., Palladium catalysed copolymerisation of ethene with alkylacrylates: polar comonomer built into the linear polymer chain. Chem.Comm.2002 , 7, 744-745.

7. Nakamura, A.; Anselment, T. M. J.; Claverie, J.; Goodall, B.; Jordan, R. F.; Mecking, S.; Rieger, B.; Sen, A.; van Leeuwen, P. W. N. M.; Nozaki, K., Ortho-Phosphinobenzenesulfonate: A Superb Ligand for Palladium-Catalyzed Coordination-Insertion Copolymerization of Polar Vinyl Monomers. Acc. Chem. Res. $\mathbf{2 0 1 3}, 46,1438-1449$.

8. Carrow, B. P.; Nozaki, K., Synthesis of Functional Polyolefins Using Cationic Bisphosphine MonoxidePalladium Complexes. J. Am. Chem. Soc. 2012, 134, 8802-8805.

9. Sui, X.; Dai, S.; Chen, C., Ethylene Polymerization and Copolymerization with Polar Monomers by Cationic Phosphine Phosphonic Amide Palladium Complexes. ACS Catal. 2015 , 5 , 5932-5937.

10. Nakano, R.; Nozaki, K., Copolymerization of Propylene and Polar Monomers Using Pd/IzQO Catalysts. J. Am. Chem. Soc.2015, 137, 10934-10937.

11. Xin, B. S.; Sato, N.; Tanna, A.; Oishi, Y.; Konishi, Y.; Shimizu, F., Nickel Catalyzed Copolymerization of Ethylene and Alkyl Acrylates.J. Am. Chem. Soc. 2017, 139, 3611-3614.

12. Chen, M.; Chen, C., A Versatile Ligand Platform for Palladium- and Nickel-Catalyzed Ethylene Copolymerization with Polar Monomers.Angew. Chem. Int. Ed. 2018 , 57, 3094-3098.

13. Mitsushige, Y.; Yasuda, H.; Carrow, B. P.; Ito, S.; Kobayashi, M.; Tayano, T.; Watanabe, Y.; Okuno, Y.; Hayashi, S.; Kuroda, J.; Okumura, Y.; Nozaki, K., Methylene-Bridged Bisphosphine Monoxide Ligands for Palladium-Catalyzed Copolymerization of Ethylene and Polar Monomers.ACS Macro Lett. 2018 , 7 , 305-311.

14. Fu, X.; Zhang, L.; Tanaka, R.; Shiono, T.; Cai, Z., Highly Robust Nickel Catalysts Containing Anilinonaphthoquinone Ligand for Copolymerization of Ethylene and Polar Monomers. Macromolecules 2017 , 50 , 9216-9221.

15. Zhang, W.; Waddell, P. M.; Tiedemann, M. A.; Padilla, C. E.; Mei, J.; Chen, L.; Carrow, B. P., Electron-Rich Metal Cations Enable Synthesis of High Molecular Weight, Linear Functional Polyethylenes.J. Am. Chem. Soc. 2018, $140,8841-8850$.

16. Michalak, A.; Ziegler, T., DFT Studies on the Copolymerization of $\alpha$-Olefins with Polar Monomers: Comonomer Binding by Nickel- and Palladium-Based Catalysts with Brookhart and Grubbs Ligands.Organometallics 2001, 20 , 1521-1532.

17. Nakano, R.; Chung, L. W.; Watanabe, Y.; Okuno, Y.; Okumura, Y.; Ito, S.; Morokuma, K.; Nozaki, K., Elucidating the Key Role of Phosphine-Sulfonate Ligands in Palladium-Catalyzed Ethylene Polymerization: Effect of Ligand Structure on the Molecular Weight and Linearity of Polyethylene. ACS Catal. 2016 , 6 , 6101-6113.

18. Rezabal, E.; Ugalde, J. M.; Frenking, G., The trans Effect in Palladium Phosphine Sulfonate Complexes. J. Phys. Chem. A2017, 121, 7709-7716. 
19. Wimmer, F. P.; Caporaso, L.; Cavallo, L.; Mecking, S.; Falivene, L., Mechanism of Insertion Polymerization of Allyl Ethers.Macromolecules 2018 , 51 , 4525-4531.

20. Nozaki, K.; Kusumoto, S.; Noda, S.; Kochi, T.; Chung, L. W.; Morokuma, K., Why Did Incorporation of Acrylonitrile to a Linear Polyethylene Become Possible? Comparison of Phosphine-Sulfonate Ligand with Diphosphine and Imine-Phenolate Ligands in the Pd-Catalyzed Ethylene/Acrylonitrile Copolymerization. J. Am. Chem. Soc.2010, 132, 16030-16042.

21. Li, M.; Wang, X.; Luo, Y.; Chen, C., A Second-Coordination-Sphere Strategy to Modulate Nickel- and Palladium-Catalyzed Olefin Polymerization and Copolymerization. Angew. Chem. Int. Ed.2017, 56, 11604-11609.

22. Sun, J.; Chen, M.; Luo, G.; Chen, C.;Luo, Y., Diphosphazane-monoxide and Phosphinesulfonate Palladium Catalyzed Ethylene Copolymerization with Polar Monomers: A Computational Study. Organometallics 2019, $38,638-646$.

23. Quintal, M. M.; Karton, A.; Iron, M. A.; Boese, A. D.; Martin, J. M. L., Benchmark Study of DFT Functionals for Late-Transition-Metal Reactions. J. Phys. Chem. A 2006 , 110 , 709-716.

24. Dohm, S.; Hansen, A.; Steinmetz, M.; Grimme, S.; Checinski, M. P., Comprehensive Thermochemical Benchmark Set of Realistic Closed-Shell Metal Organic Reactions. J. Chem. Theory Comput. 2018 ,14, 2596-2608.

25. Shamov, G. A.; Budzelaar, P. H. M.; Schreckenbach, G., Performance of the Empirical Dispersion Corrections to Density Functional Theory: Thermodynamics of Hydrocarbon Isomerizations and Olefin Monomer Insertion Reactions. J. Chem. Theory Comput. 2010 ,6 , 477-490.

26. Castro, L.; Kirillov, E.; Miserque, O.; Welle, A.; Haspeslagh, L.; Carpentier, J.-F.; Maron, L., Are Solvent and Dispersion Effects Crucial in Olefin Polymerization DFT Calculations? Some Insights from Propylene Coordination and Insertion Reactions with Group 3 and 4 Metallocenes.ACS Catal. 2015 , 5 , 416-425.

27. Kang, M.; Sen, A.; Zakharov, L.; Rheingold, A. L., Diametrically Opposite Trends in Alkene Insertion in Late and Early Transition Metal Compounds: Relevance to Transition-Metal-Catalyzed Polymerization of Polar Vinyl Monomers. J. Am. Chem. Soc. 2002 ,124, 12080-12081.

28. Frisch, M. J.; Trucks, G. W.; Schlegel, H. B.; Scuseria, G. E.; Robb, M. A.; Cheeseman, J. R.; Scalmani, G.; Barone, V.; Petersson, G. A.; Nakatsuji, H.; Li, X.; Caricato, M.; Marenich, A. V.; Bloino, J.; Janesko, B. G.; Gomperts, R.; Mennucci, B.; Hratchian, H. P.; Ortiz, J. V.; Izmaylov, A. F.; Sonnenberg, J. L.; Williams; Ding, F.; Lipparini, F.; Egidi, F.; Goings, J.; Peng, B.; Petrone, A.; Henderson, T.; Ranasinghe, D.; Zakrzewski, V. G.; Gao, J.; Rega, N.; Zheng, G.; Liang, W.; Hada, M.; Ehara, M.; Toyota, K.; Fukuda, R.; Hasegawa, J.; Ishida, M.; Nakajima, T.; Honda, Y.; Kitao, O.; Nakai, H.; Vreven, T.; Throssell, K.; Montgomery Jr., J. A.; Peralta, J. E.; Ogliaro, F.; Bearpark, M. J.; Heyd, J. J.; Brothers, E. N.; Kudin, K. N.; Staroverov, V. N.; Keith, T. A.; Kobayashi, R.; Normand, J.; Raghavachari, K.; Rendell, A. P.; Burant, J. C.; Iyengar, S. S.; Tomasi, J.; Cossi, M.; Millam, J. M.; Klene, M.; Adamo, C.; Cammi, R.; Ochterski, J. W.; Martin, R. L.; Morokuma, K.; Farkas, O.; Foresman, J. B.; Fox, D. J.Gaussian 16 Rev. C.01 , Wallingford, CT, 2016.

29. Grimme, S., Semiempirical GGA-type density functional constructed with a long-range dispersion correction. J. Comput. Chem.2006 , 27, 1787-1799.

30. Grimme, S.; Antony, J.; Ehrlich, S.; Krieg, H., A consistent and accurate ab initio parametrization of density functional dispersion correction (DFT-D) for the 94 elements H-Pu. J. Chem. Phys.2010 , 132 , 154104 .

31. Grimme, S.; Ehrlich, S.; Goerigk, L., Effect of the damping function in dispersion corrected density functional theory. J. Comput. Chem. 2011, 32, 1456-1465. 
32. Hay, P. J.; Wadt, W. R., Ab initio effective core potentials for molecular calculations. Potentials for K to Au including the outermost core orbitals. J. Chem. Phys. 1985, 82 , 299-310.

33. Andrae, D.; Häussermann, U.; Dolg, M.; Stoll, H.; Preuss, Energy-adjustedab initio pseudopotentials for the second and third row transition elements. Theor. Chim. Acta 1990 , 77, 123-141.

34. Cossi, M.; Rega, N.; Scalmani, G.; Barone, V., Energies, structures, and electronic properties of molecules in solution with the C-PCM solvation model. J. Comput. Chem. 2003 , 24, 669-681.

35. Marenich, A. V.; Cramer, C. J.; Truhlar, D. G., Universal Solvation Model Based on Solute Electron Density and on a Continuum Model of the Solvent Defined by the Bulk Dielectric Constant and Atomic Surface Tensions. J. Phys. Chem. B 2009 , 113, 6378-6396.

36. Becke, A. D., Density-functional exchange-energy approximation with correct asymptotic behavior. Phys.Rev. A $1988,38,3098-3100$.

37. Perdew, J. P., Density-functional approximation for the correlation energy of the inhomogeneous electron gas. Phys. Rev. B1986, $33,8822-8824$.

38. Grimme, S., Semiempirical GGA-type density functional constructed with a long-range dispersion correction. J. Comput. Chem.2006 , 27, 1787-1799.

39. Perdew, J. P.; Chevary, J. A.; Vosko, S. H.; Jackson, K. A.; Pederson, M. R.; Singh, D. J.; Fiolhais, C., Atoms, molecules, solids, and surfaces: Applications of the generalized gradient approximation for exchange and correlation. Phys. Rev. B 1992, $46,6671-6687$.

40. Perdew, J. P.; Burke, K.; Ernzerhof, M., Generalized Gradient Approximation Made Simple. Phys. Rev. Lett. $1996,777,3865-3868$.

41. Hamprecht, F. A.; Cohen, A. J.; Tozer, D. J.; Handy, N. C., Development and assessment of new exchange-correlation functionals.J. Chem. Phys. 1998, $109,6264-6271$.

42. Boese, A. D.; Doltsinis, N. L.; Handy, N. C.; Sprik, M., New generalized gradient approximation functionals. J. Chem. Phys.2000, 112, 1670-1678.

43. Boese, A. D.; Handy, N. C., A new parametrization of exchange-correlation generalized gradient approximation functionals. J. Chem. Phys. 2001, 114, 5497-5503.

44. Tao, J.; Perdew, J. P.; Staroverov, V. N.; Scuseria, G. E., Climbing the density functional ladder: nonempirical meta-generalized gradient approximation designed for molecules and solids. Phys. Rev. Lett.2003, 91,146401 .

45. Zhao, Y.; Truhlar, D. G., A new local density functional for main-group thermochemistry, transition metal bonding, thermochemical kinetics, and noncovalent interactions. J. Chem. Phys.2006 , 125 , 194101.

46. Boese, A. D.; Handy, N. C., New exchange-correlation density functionals: The role of the kinetic-energy density. J. Chem. Phys. 2002 , $116,9559-9569$.

47. Zhao, Y.; Truhlar, D. G., The M06 suite of density functionals for main group thermochemistry, thermochemical kinetics, noncovalent interactions, excited states, and transition elements: two new functionals and systematic testing of four M06-class functionals and 12 other functionals. Theor. Chem. Acc. 2008, $120,215-241$.

48. Grimme, S., Semiempirical hybrid density functional with perturbative second-order correlation. $J$. Chem. Phys.2006 , $124,034108$.

49. Stephens, P. J.; Devlin, F. J.; Chabalowski, C. F.; Frisch, M. J., Ab Initio Calculation of Vibrational Absorption and Circular Dichroism Spectra Using Density Functional Force Fields. J. Phys. Chem.1994, $98,11623-11627$. 
50. Becke, A. D., Density-functional thermochemistry. III. The role of exact exchange. J. Chem. Phys. $1993,98,5648-5652$.

51. Perdew, J. P.; Wang, Y., Accurate and simple analytic representation of the electron-gas correlation energy. Phys. Rev. B1992, 45, 13244-13249.

52. Becke, A. D., Densityy., Accurate and simple analytic representation of the electron-gas correlation energy. ra Using Density FunctionalJ. Chem. Phys. 1996, 104, 1040-1046.

53. Adamo, C.; Barone, V., Exchange functionals with improved long-range behavior and adiabatic connection methods without adjustable parameters: The mPW and mPW1PW models. J. Chem. Phys. 1998 ,108, 664-675.

54. Schmider, H. L.; Becke, A. D., Optimized density functionals from the extended G2 test set. J. Chem. Phys. $1998,108,9624-9631$.

55. Lee, C.; Yang, W.; Parr, R. G., Development of the Colle-Salvetti correlation-energy formula into a functional of the electron density.Phys.Rev. B 1988, 37, 785-789.

56. Wilson, P. J.; Bradley, T. J.; Tozer, D. J., Hybrid exchange-correlation functional determined from thermochemical data and ab initio potentials. J. Chem. Phys. 2001, 115, 9233-9242.

57. Adamo, C.; Barone, V., Toward reliable density functional methods without adjustable parameters: The PBE0 model. J. Chem. Phys.1999 , 110 , 6158-6170.

58. Adamo, C.; Barone, V., Toward reliable adiabatic connection models free from adjustable parameters. Chem.Phys. Lett. $1997,274,242-250$.

59. Cohen, A. J.; Handy, N. C., Dynamic correlation. Mol. Phys.2001 , 99 , 607-615.

60. Heyd, J.; Scuseria, G. E., Efficient hybrid density functional calculations in solids: assessment of the Heyd-Scuseria-Ernzerhof screened Coulomb hybrid functional. J. Chem. Phys. 2004 ,121, 1187-1192.

61. Krukau, A. V.; Vydrov, O. A.; Izmaylov, A. F.; Scuseria, G. E., Influence of the exchange screening parameter on the performance of screened hybrid functionals. J. Chem. Phys. 2006 ,125, 224106.

62. Chai, J.-D.; Head-Gordon, M., Long-range corrected hybrid density functionals with damped atom-atom dispersion corrections. Phys. Chem. Chem. Phys. 2008, 10, 6615-6620.

63. Chai, J. D.; Head-Gordon, M., Systematic optimization of long-range corrected hybrid density functionals. J. Chem. Phys.2008, 128 (8), 084106.

64. Staroverov, V. N.; Scuseria, G. E.; Tao, J.; Perdew, J. P., Comparative assessment of a new nonempirical density functional: Molecules and hydrogen-bonded complexes. J. Chem. Phys.2003, 119 , 12129-12137.

65. Xu, X.; Goddard, W. A., The X3LYP extended density functional for accurate descriptions of nonbond interactions, spin states, and thermochemical properties. Proc. Natl. Acad. Sci. USA2004, 101 , 2673.

66. Vydrov, O. A.; Heyd, J.; Krukau, A. V.; Scuseria, G. E., Importance of short-range versus long-range Hartree-Fock exchange for the performance of hybrid density functionals. J. Chem. Phys.2006 , 125, 074106 .

67. Vydrov, O. A.; Scuseria, G. E., Assessment of a long-range corrected hybrid functional. J. Chem. Phys. 2006, 125, 234109 .

68. Winne, J. M.; Catak, S.; Waroquier, M.; Van Speybroeck, V., Scope and mechanism of the $(4+3)$ cycloaddition reaction of furfuryl cations.Angew. Chem. Int. Ed. 2011, 50 , 11990-11993.

69. Boese, A. D.; Martin, J. M., Development of density functionals for thermochemical kinetics. J. Chem. Phys. $2004,121,3405-3416$. 
70. Becke, A. D., A new mixing of Hartreeent of density functionals for thermochemical. J. Chem. Phys. $1993,98,1372-1377$.

71. Zhao, Y.; Truhlar, D. G., Comparative DFT Study of van der Waals Complexes: Rare-Gas Dimers, Alkaline-Earth Dimers, Zinc Dimer, and Zinc-Rare-Gas Dimers. J. Phys. Chem. A 2006 , 110 , 5121-5129.

72. Zhao, Y.; Truhlar, D. G., Density Functional for Spectroscopy: No Long-Range Self-Interaction Error, Good Performance for Rydberg and Charge-Transfer States, and Better Performance on Average than B3LYP for Ground States. J. Phys. Chem. A 2006 , 110 , 13126-13130.

73. Zhao, Y.; Schultz, N. E.; Truhlar, D. G., Design of Density Functionals by Combining the Method of Constraint Satisfaction with Parametrization for Thermochemistry, Thermochemical Kinetics, and Noncovalent Interactions. J. Chem. Theory Comput. 2006 ,2 , 364-382.

74. Zhao, Y.; Schultz, N. E.; Truhlar, D. G., Exchange-correlation functional with broad accuracy for metallic and nonmetallic compounds, kinetics, and noncovalent interactions. J. Chem. Phys.2005, 123, 161103.

75. Schenker, S.; Schneider, C.; Tsogoeva, S. B.; Clark, T., Assessment of Popular DFT and Semiempirical Molecular Orbital Techniques for Calculating Relative Transition State Energies and Kinetic Product Distributions in Enantioselective Organocatalytic Reactions. J. Chem. Theory Comput. 2011 , 7 , 3586-3595.

Table of Content Use Only

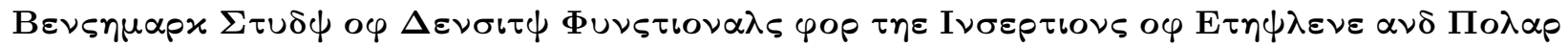

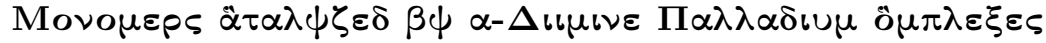

Ce Liang, ${ }^{+}$Jimin Yang, ${ }^{+}$Gen Luo, ${ }^{\S}$ Yi Luo $^{+, *}$ 